\title{
Numerical Solution of a Two-asset Option Valuation PDE by ADI Finite Difference Discretization
}

\author{
Karel in 't Hout and Radoslav Valkov \\ Department of Mathematics and Computer Science, University of Antwerp, Middelheimlaan 1, B-2020 Antwerp, \\ Belgium. E-mail: \{karel.inthout, radoslav.valkov\} Quantwerpen.be
}

\begin{abstract}
The numerical valuation of European two-asset options is considered under the Black-Scholes model. A numerical method is discussed based on FD discretization of the pertinent PDE on nonuniform grids and subsequent discretization in time by ADI schemes. We investigate stability and convergence experimentally for both the option value function and the Cross Gamma function, which is important to nonlinear extensions of the model, beyond the standard Black-Scholes framework.
\end{abstract}

Keywords: financial option valuation, convection-diffusion problems, two-asset option, finite difference methods, ADI schemes PACS: $02.60 . \mathrm{Lj}, 02.70 . \mathrm{Bf}, 89.65 . \mathrm{Gh}$

\section{TWO-ASSET OPTION VALUATION PDE}

This note deals with the numerical solution of a partial differential equation (PDE) for the valuation of European-style options on two underlying assets,

$$
\frac{\partial u}{\partial t}=\frac{1}{2} \sigma_{1}^{2} s_{1}^{2} \frac{\partial^{2} u}{\partial s_{1}^{2}}+\rho \sigma_{1} \sigma_{2} s_{1} s_{2} \frac{\partial^{2} u}{\partial s_{1} \partial s_{2}}+\frac{1}{2} \sigma_{2}^{2} s_{2}^{2} \frac{\partial^{2} u}{\partial s_{2}^{2}}+r s_{1} \frac{\partial u}{\partial s_{1}}+r s_{2} \frac{\partial u}{\partial s_{2}}-r u \quad\left(s_{1}>0, s_{2}>0,0<t \leq T\right) .
$$

Here $u\left(s_{1}, s_{2}, t\right)$ represents the fair value of the option if at time $T-t$ the two underlying asset prices equal $s_{1}$ and $s_{2}$ where $T$ is the given maturity time of the option. The parameters $\sigma_{1}, \sigma_{2}>0$ are the volatilities of the underlying asset price processes and $\rho \in[-1,1]$ denotes their correlation. Further, $r$ is the risk-free interest rate. The PDE (1) forms a well-known generalization of the reputed Black-Scholes PDE. Clearly, it can be viewed as a time-dependent convection-diffusion-reaction equation on an unbounded two-dimensional spatial domain. Note that a mixed spatial derivative term is present in the case $\rho \neq 0$.

We shall consider here a European butterfly option on the maximum of the two asset prices. A butterfly option can be regarded as a combination of two long calls with strikes $K_{1}$ and $K_{2}$ and two short calls both with strike $K=\left(K_{1}+K_{2}\right) / 2$. Thus, for given values $K_{1}, K_{2}>0$ with $K_{1}<K_{2}$, the initial condition is

$$
u\left(s_{1}, s_{2}, 0\right)=\max \left(s-K_{1}, 0\right)-2 \max (s-K, 0)+\max \left(s-K_{2}, 0\right) \quad \text { with } s=\max \left(s_{1}, s_{2}\right) \quad\left(s_{1}>0, s_{2}>0\right) .
$$

Observe that the initial function is nonsmooth: it is continuous, but not everywhere differentiable on its domain. The boundary conditions are obtained by inserting $s_{1}=0$ and $s_{2}=0$ into (1), i.e., the classical Black-Scholes PDE is satisfied at these two boundaries. In addition to the option value function, also the hedging quantities, often called the Greeks, are of main importance in practice. Here we consider the Cross Gamma of the option,

$$
\Gamma=\frac{\partial^{2} u}{\partial s_{1} \partial s_{2}} .
$$

The Cross Gamma is of particular interest in view of nonlinear extensions of (1) that arise when incorporating e.g. transaction costs into the model, which is our objective for future research. In the present note our aim is to validate the proposed numerical discretization method in the case of the linear PDE (1), for which an exact solution in semi-closed analytical form is at hand, cf. e.g. [6].

\section{DISCRETIZATION METHOD}

For the numerical solution of the initial-boundary value problem for (1) we adopt the well-known method-of-lines approach, whereby first a discretization in the $s_{1}, s_{2}$ variables is performed and next a discretization in time $t$. We

Proceedings of the International Conference on Numerical Analysis and Applied Mathematics 2014 (ICNAAM-2014)

AIP Conf. Proc. 1648, 020007-1-020007-4; doi: 10.1063/1.4912311

(C) 2015 AIP Publishing LLC 978-0-7354-1287-3/\$30.00 
construct the discretization in $s_{1}, s_{2}$ by finite difference (FD) formulas on nonuniform Cartesian grids. Nonuniform grids are often preferable over uniform grids, both for numerical and financial reasons. First, the unbounded $\left(s_{1}, s_{2}\right)$ domain is truncated to a bounded set $\left[0, S_{\max }\right] \times\left[0, S_{\max }\right]$ with fixed $S_{\max }$ taken sufficiently large. At $s_{1}=S_{\max }$ and $s_{2}=S_{\max }$ additional boundary conditions are provided. For the butterfly option under consideration, a natural choice is given by homogeneous Dirichlet conditions, since the option value will be close to zero whenever an underlying asset price is large. The meshes in the $s_{1}$ - and $s_{2}$-directions are the same and defined such that they are uniform in the interval $\left[\frac{1}{2} K_{1}, \frac{3}{2} K_{2}\right]$ and nonuniform outside, with mesh widths that smoothly increase when moving away from this interval. On the obtained Cartesian grid, all derivatives on the right-hand side of equation (1) are then replaced by corresponding second-order central FD formulas. For the sake of brevity, we omit the details of the grid and the FD formulas here, but cf. e.g. [1]. To alleviate the adverse impact of the nonsmoothness of the initial function (2) on the accuracy of the discretization, we follow [7] and replace the value of the initial function by a cell average at those grid points that lie closest to the points of nondifferentiability. After the discretization in the $s_{1}, s_{2}$ variables one arrives at a large system of stiff ordinary differential equations,

$$
U^{\prime}(t)=A U(t) \quad(0 \leq t \leq T), \quad U(0)=U_{0}
$$

Here $A$ is a given real square matrix, $U_{0}$ is a given real vector and the entries of the solution vector $U(t)$ represent approximations to the option values $u\left(s_{1}, s_{2}, t\right)$ at the $\left(s_{1}, s_{2}\right)$-grid points, ordered in a convenient way. The vector $U_{0}$ is directly obtained from the initial condition (2) and taking into account the above cell average modification.

For the discretization in time of (3) we consider splitting schemes of the Alternating Direction Implicit (ADI) type. To this purpose, let $A$ be decomposed as $A=A_{0}+A_{1}+A_{2}$ where the matrix $A_{0}$ corresponds to the mixed derivative term in (1) and for $j=1,2$ the matrix $A_{j}$ corresponds to all derivatives in the $s_{j}$-direction. Let $\theta>0$ be a given real parameter and time step $\Delta t=T / N$ with integer $N \geq 1$. Set $t_{n}=n \Delta t$. The following three popular ADI schemes generate, in a one-step manner, approximations $U_{n}$ to the solution vectors $U\left(t_{n}\right)$ of (3) successively for $n=1,2, \ldots, N$.

Craig-Sneyd (CS) scheme:

$$
\left\{\begin{array}{l}
Y_{0}=U_{n-1}+\Delta t A U_{n-1} \\
Y_{j}=Y_{j-1}+\theta \Delta t A_{j}\left(Y_{j}-U_{n-1}\right) \quad(j=1,2) \\
\widetilde{Y}_{0}=Y_{0}+\frac{1}{2} \Delta t A_{0}\left(Y_{2}-U_{n-1}\right) \\
\widetilde{Y}_{j}=\widetilde{Y}_{j-1}+\theta \Delta t A_{j}\left(\widetilde{Y}_{j}-U_{n-1}\right) \quad(j=1,2) \\
U_{n}=\widetilde{Y}_{2}
\end{array}\right.
$$

Modified Craig-Sneyd (MCS) scheme:

$$
\left\{\begin{array}{l}
Y_{0}=U_{n-1}+\Delta t A U_{n-1}, \\
Y_{j}=Y_{j-1}+\theta \Delta t A_{j}\left(Y_{j}-U_{n-1}\right) \quad(j=1,2), \\
\widehat{Y}_{0}=Y_{0}+\theta \Delta t A_{0}\left(Y_{2}-U_{n-1}\right), \\
\widetilde{Y}_{0}=\widehat{Y}_{0}+\left(\frac{1}{2}-\theta\right) \Delta t A\left(Y_{2}-U_{n-1}\right), \\
\widetilde{Y}_{j}=\widetilde{Y}_{j-1}+\theta \Delta t A_{j}\left(\widetilde{Y}_{j}-U_{n-1}\right) \quad(j=1,2), \\
U_{n}=\widetilde{Y}_{2} .
\end{array}\right.
$$

Hundsdorfer-Verwer (HV) scheme:

$$
\left\{\begin{array}{l}
Y_{0}=U_{n-1}+\Delta t A U_{n-1} \\
Y_{j}=Y_{j-1}+\theta \Delta t A_{j}\left(Y_{j}-U_{n-1}\right) \quad(j=1,2) \\
\widetilde{Y}_{0}=Y_{0}+\frac{1}{2} \Delta t A\left(Y_{2}-U_{n-1}\right) \\
\widetilde{Y}_{j}=\widetilde{Y}_{j-1}+\theta \Delta t A_{j}\left(\widetilde{Y}_{j}-Y_{2}\right) \quad(j=1,2) \\
U_{n}=\widetilde{Y}_{2}
\end{array}\right.
$$


The CS scheme has classical (nonstiff) order of consistency two if and only if $\theta=\frac{1}{2}$. For the MCS and HV schemes this order is equal to two for any given $\theta$. In all three ADI schemes the $A_{0}$ part is always treated in an explicit fashion and the $A_{1}, A_{2}$ parts are treated in an implicit fashion. In every time step, four linear systems of equations need to be solved, each involving either $A_{1}$ or $A_{2}$. Since the pertinent matrices are essentially tridiagonal, this can be done efficiently using $L U$ factorization. Accordingly, for each ADI scheme, the number of floating point operations per time step is directly proportional to the number of $\left(s_{1}, s_{2}\right)$-grid points, which is highly favorable.

Recently, various unconditional stability results in the von Neumann sense have been derived for the ADI schemes (4), (5), (6) relevant to multidimensional convection-diffusion equations with mixed derivative terms, see [2, 3, 4, 5]. Based on this, we select here $\theta=\frac{1}{2}, \theta=\frac{1}{3}$ and $\theta=\frac{1}{2}+\frac{1}{6} \sqrt{3}$ for the CS, MCS and HV schemes, respectively.

\section{NUMERICAL EXPERIMENTS}
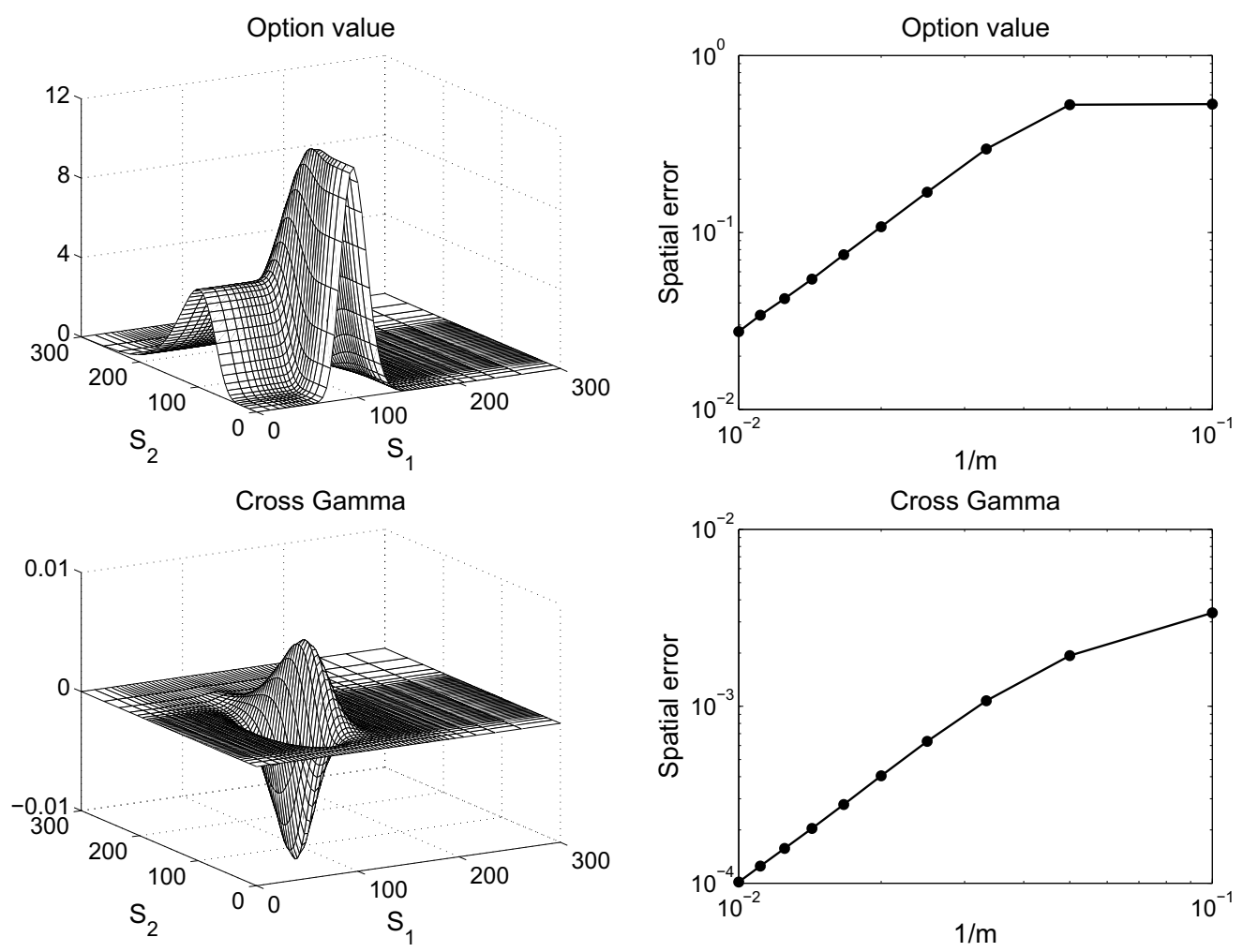

FIGURE 1. Left column: graphs of option value function and Cross Gamma function for European butterfly option on the maximum of two asset prices. Right column: spatial discretization errors for $m=10,20, \ldots, 100$. Parameters given by (7).

For the numerical experiment we consider the following parameter values regarding the European butterfly option, the underlying asset pricing model and the domain truncation:

$$
T=1, K_{1}=80, K_{2}=120, \sigma_{1}=0.1, \sigma_{2}=0.3, \rho=-0.5, r=0.05, \quad S_{\max }=300 .
$$

The left column of Figure 1 shows the graphs of the butterfly option value function $u$ and the corresponding Cross Gamma function $\Gamma$ when $t=T$ obtained by the above numerical discretization method using $m=N=50$, where $m$ denotes the number of points in the $s_{1}$ (or $s_{2}$ ) mesh. Here $\Gamma$ has been approximated by applying the pertinent second-order central FD formula employed in the discretization method for (1) to the approximated $u$-values.

We first examine the convergence behavior of the semidiscretization (3) as a function of $m$. The right column of Figure 1 displays for $m=10,20, \ldots, 100$ the maximum error of the approximations to the exact $u$-and $\Gamma$-values at $t=T$ where $N=100$ is taken such that the temporal error is negligible. One readily observes that (ignoring $m=10,20$ ) the convergence order is equal to two, both in the case of $u$ and $\Gamma$, which is as desired. 

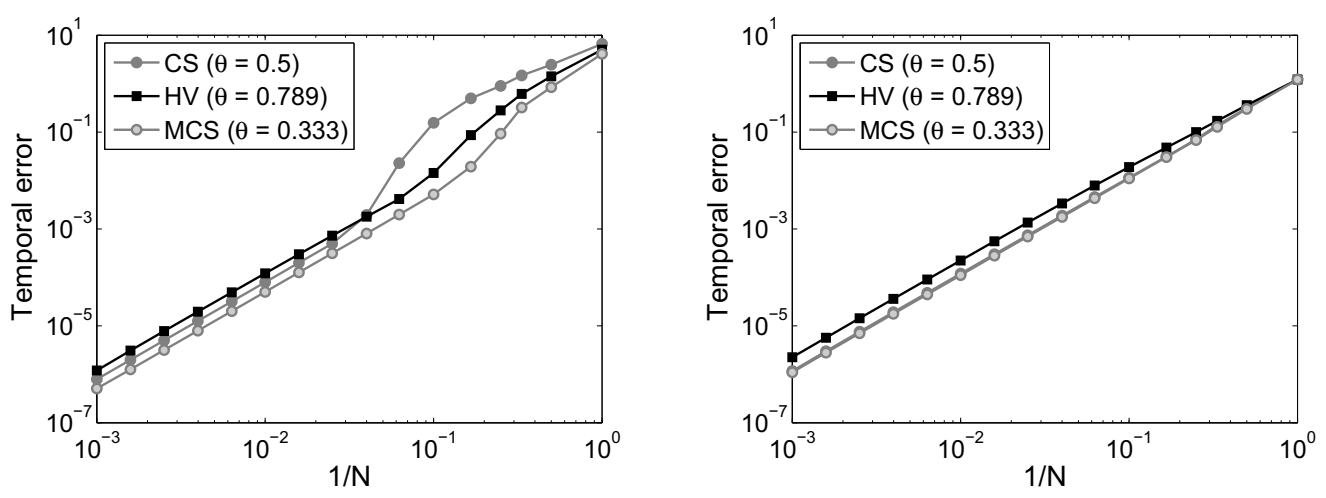

FIGURE 2. Temporal discretization errors for the CS, MCS and HV schemes when $m=100$ without damping (left) and with damping (right). Parameters given by (7).

We next study the temporal convergence behavior of each of the three ADI schemes (4), (5), (6) with $\theta$ specified above. Thus, the obtained approximations at $t=T$ are compared to $U(T)$ defined by (3). For the latter, a reference solution is computed using the HV scheme with $N=5000$ time steps. The left picture in Figure 2 displays for $m=100$ and a set of values $N$ with $1 \leq N \leq 1000$ the maximum error of the approximations to $U(T)$. Clearly, the errors are all bounded from above by a moderate constant and decrease monotonically as $N$ increases, which indicates an unconditionally stable behavior of the three ADI schemes. Next, for each scheme, the observed temporal convergence order is equal to two, ignoring small $N$. Note that for the CS scheme the errors are relatively large whenever the number of time steps $N$ is small to modest. For the MCS and HV schemes this undesirable behavior is much less present. The cause for this (well-known) phenomenon lies in the nonsmooth initial function (2) and the different damping properties of the three schemes. A remedy is to replace the first time step, at $t=0$, by two half steps using the backward Euler method. This is often called Rannacher time stepping. The right picture of Figure 2 shows the obtained result. Clearly, the convergence behavior is now regular and a temporal convergence order equal to two is retained, at the expense of a modest increase in the errors for large $N$.

Additional experiments for other parameter sets and other values $m$ lead to the same favorable conclusions. In future research we shall adapt and investigate the presented discretization method for contemporary nonlinear versions of (1).

\section{ACKNOWLEDGEMENTS}

This work was supported by the European Union in the FP7-PEOPLE-2012-ITN Program under Grant Agreement Number 304617 (FP7 Marie Curie Action, Project Multi-ITN STRIKE - Novel Methods in Computational Finance).

\section{REFERENCES}

1. T. Haentjens \& K. J. in 't Hout, Alternating direction implicit finite difference schemes for the Heston-Hull-White PDE, J. Comp. Finan. 16 (2012) 83-110.

2. K. J. in 't Hout \& C. Mishra, Stability of the modified Craig-Sneyd scheme for two-dimensional convection-diffusion equations with mixed derivative term, Math. Comp. Simul. 81 (2011) 2540-2548.

3. K. J. in 't Hout \& C. Mishra, Stability of ADI schemes for multidimensional diffusion equations with mixed derivative terms, Appl. Numer. Math. 74 (2013) 83-94.

4. K. J. in 't Hout \& B. D. Welfert, Stability of ADI schemes applied to convection-diffusion equations with mixed derivative terms, Appl. Numer. Math. 57 (2007) 19-35.

5. K. J. in 't Hout \& B. D. Welfert, Unconditional stability of second-order ADI schemes applied to multi-dimensional diffusion equations with mixed derivative terms, Appl. Numer. Math. 59 (2009) 677-692.

6. R. M. Stulz, Options on the minimum or the maximum of two risky assets, J. Finan. Econ. 10 (1982) 161-185.

7. D. Tavella \& C. Randall, Pricing Financial Instruments, Wiley, New York, 2000. 
AIP Conference Proceedings is copyrighted by AIP Publishing LLC (AIP). Reuse of AIP content is subject to the terms at: http://scitation.aip.org/termsconditions. For more information, see http://publishing.aip.org/authors/rights-and-permissions. 